\title{
Compensatory effects in the PI3K/PTEN/AKT signalling network following receptor tyrosine kinase inhibition
}

Alexey Goltsov ${ }^{\mathrm{a} *}$, Dana Faratian ${ }^{\mathrm{b}}$, Simon P Langdon ${ }^{\mathrm{b}}$, James Bown ${ }^{\mathrm{a}}$, Igor Goryanin ${ }^{\mathrm{c}}$ and David J Harrison $^{\mathrm{b}}$

${ }^{a}$ Centre for Research in Informatics and Systems Pathology, University of Abertay Dundee, Dundee, DD1 1HG, United Kingdom

${ }^{\mathrm{b}}$ Edinburgh Breakthrough Research Unit and Division of Pathology, Institute of Genetics and Molecular Medicine, University of Edinburgh, Edinburgh, EH4 2XR, United Kingdom

${ }^{\mathrm{c}}$ School of Informatics, University of Edinburgh, Edinburgh, EH8 9AB, United Kingdom

* Corresponding author: Alexey Goltsov

Address: SIMBIOS, the University of Abertay Dundee, Dundee, DD1 1HG, UK

Tel: +441382308471

E-mail: a.goltsov@abertay.ac.uk

Keywords: HER2 inhibitor; pertuzumab; ERK/PI3K/PTEN/AKT signalling network; anticancer drug resistance. 


\begin{abstract}
Overcoming de novo and acquired resistance to anticancer drugs that target signalling networks is a formidable challenge for drug design and effective cancer therapy.

Understanding the mechanisms by which this resistance arises may offer a route to addressing the insensitivity of signalling networks to drug intervention and restore the efficacy of anticancer therapy. Extending our recent work identifying PTEN as a key regulator of herceptin sensitivity, we present an integrated theoretical and experimental approach to study the compensatory mechanisms within the PI3K/PTEN/AKT signalling network that afford resistance to receptor tyrosine kinase (RTK) inhibition by anti-HER2 monoclonal antibodies. In a computational model representing the dynamics of the signalling network, we define a single control parameter that encapsulates the balance of activities of the enzymes involved in the PI3K/PTEN/AKT cycle. By varying this control parameter we are able to demonstrate both distinct dynamic regimes of behaviour of the signalling network and the transitions between those regimes. We demonstrate resistance, sensitivity, and suppression of RTK signals by the signalling network. Through model analysis we link the sensitivity-toresistance transition to specific compensatory mechanisms within our signalling network. We study this transition in detail theoretically by variation of activities of PTEN, PI3K, AKT enzymes, and use the results to inform experiments that perturb the signalling network using combinatorial inhibition of RTK, PTEN, and PI3K enzymes in human ovarian carcinoma cell lines. We find good alignment between theoretical predictions and experimental results. We discuss the application of the results to the challenges of hypersensitivity of the signalling network to RTK signals, suppression of drug resistance, and efficacy of drug combinations in anticancer therapy.
\end{abstract}




\section{Introduction}

Extensive experimental and theoretical studies of the architecture and dynamics of signalling networks (SNs) [1-7] have revealed that the complexity of these networks endows cells with marked sensitivity to external receptor signals along with robustness in signal transduction and processing [8-10]. This sensitivity provides the cell with a well-defined output signal in response to external input stimuli against a background of multiple, parallel processes in linked signal cascades, while robustness secures unambiguous concordance between output and input signals in the face of perturbations and noise that inevitably occur in living processes. This combination of properties arises from the unique protein interactomes, self-assembling of signalling protein complexes, protein scaffolding, and multi-level control throughout signal transduction from membrane receptors to nucleus.

The properties of sensitivity and robustness are not determined by any one part of the SNs but emerge from the complex, system-scale behaviour of those SN. This emergent behaviour thus depends on the interactions among individual components of the SN. The functioning of those individual components may be subject to variation due to the external environment and/or internal perturbations, and these variations have the potential to impact on those emergent properties. While the cell is, in general, resilient to this underlying variation, a change in sensitivity and/or robustness may occur as a result of various adaptive processes; for example during pathogenesis of disease or acquisition of drug resistance, which itself can be regarded as an adaptation process to therapeutic drug intervention [7-13].

With particular reference to cancer, acquired and de novo resistance to anticancer drugs (also termed cancer robustness) are manifestations of the loss of SN sensitivity to external signals $[12,13]$. Resistance emerges in the case of most effective drugs, including anti-HER2 monoclonal antibodies trastuzumab and pertuzumab, which inhibit receptor tyrosine kinase (RTK) and suppress activation of the PI3K/AKT survival pathway. This resistance causes potent cell growth and reduces the impact of treatment on cancer cell mortality in HER2dependent breast and ovarian cancers $[7,14,15]$. Primary sensitivity of tumour cells to these drugs may vary dramatically for various cancer cell lines, dependent in part on the expression level of the proteins involved in SNs [13]. Many cancers lose original therapeutic responsiveness after prolonged treatment and acquire resistance to the RTK inhibitors [13]. Various mutations in SNs can cause this resistance, leading to more rapid cancer cell growth [11-13]. 
We hypothesise that sensitivity-to-resistance transition results from compensatory features inherent in SNs. We have previously shown that the tumour suppressor PTEN involved in PI3K/PTEN/AKT signalling network plays a key role in resistance to RTK inhibition [7]. Here we extend with inclusion of additional mechanisms our approach in [7] to study in detail the signalling network behaviour relating to drug resistance acquisition, with a focus on identifying the compensatory features of the PTEN/PI3K/AKT pathway. In particular, we explore the observed effect of PTEN on drug resistance [7] within the broader context of compensatory mechanisms emerging in this $\mathrm{SN}$ as a result of sensitivity-toresistance transition.

More generally, both experimental and theoretical studies have revealed several systemscale properties of SNs which can provide such compensatory mechanisms, including (i) redundancy and multiple cross-talk in SN topology, affording alternate pathways to effect a particular behaviour $[1,12,13,16]$; (ii) gene/metabolic feedback control loops due to combinations of activation and inhibition/repression processes, regulating cell response to perturbation [8]; and (iii) mutations of the drug targets leading to acquired resistance in phenotypes under particular drug regimes $[8,11,12]$.

Here, we focus on another compensatory mechanism typical in SNs, made possible due to specific components present in almost all cellular SNs, for example, in MAP kinase pathways: signalling cycles composed of two inter-convertible enzymes, kinases and phosphatases. Numerous theoretical and experimental studies of the dynamic properties of these cycles have revealed a wide spectrum of the different modes of cycle functioning, such as toggle switch, amplification, ultrasensitivity, signal transduction, and oscillation [17-22], which are realised by different sets of kinetic parameters of individual enzymes involved in SNs. Also experimental and clinical research has shown that aberrant expression and mutations of the enzymes making up those cycles cause cancer development and resistance to anticancer drugs [13]. We suggest that mutations of the enzymes involved in the signalling cycles can cause the transitions among these modes and some of these modes may underlie compensatory mechanisms for drug intervention.

We present a systems biology approach to elucidate the connection between the kinetic properties of the PI3K/PTEN kinase-phosphatase signalling cycle and the response of the whole ERK/PI3K/PTEN/AKT pathway to RTK stimuli. Using both computational and experimental methods we studied the compensatory mechanisms within the PI3K/PTEN/AKT signalling pathway that determine the change in sensitivity of AKT activation to RTK inhibition in ovarian cancer cells. In our theoretical work we defined this signalling pathway 
using kinetic modelling, and sensitivity of the SN to RTK inhibitor (pertuzumab) was investigated by perturbing the PI3K/PTEN/AKT signalling cycle. The balance among these enzyme activities was encapsulated within a control parameter that integrates the functional properties of multiple SN components. We then varied this control parameter, effecting a combination of perturbations to the relevant component properties, to represent different mutations and the impact of external inhibitors. The perturbation technique we describe allowed us to vary the SN dynamics from high to low sensitivity to RTK signals and to explore the sensitivity-to-resistance transition. We also perturbed the signalling pathway experimentally to test theoretical model predictions.

\section{Materials and methods}

\subsection{Computational methods}

A study of the compensatory properties of the SN against RTK inhibitor is based on an application of the computational model of RAF/MEK/ERK and PI3K/PTEN/AKT signalling developed in [7]. The model, its extension, and computational methods are outlined in section 2.1.1. In Section 2.1.2 we provide the method of derivation of the control parameter, which is based on the analytical analysis of a subsystem of the $\mathrm{SN}$ - the PI3K/PTEN/AKT signalling cycle.

\subsubsection{Signalling network model}

To study the compensatory properties of the $\mathrm{SN}$ we built upon our existing kinetic model of RAF/MEK/ERK and PI3K/PTEN/AKT signalling [7]. This model describes the response kinetics of the $\mathrm{SN}$ to heregulin (HRG)-induced HER3/HER2 receptor heterodimerisation and the effect of RTK inhibitor, pertuzumab (2C4 antibody) $[14,15]$ on ERK and AKT activation in the human ovarian carcinoma cell line PE04 [7]. The scheme of the signalling network that corresponds to the model is shown in Fig. 1. In the current model we omitted the formation of HER3/HER4, HER4/HER4, and HER2/HER4 receptor complexes because HER4 receptor expression was barely detectable in PE04 cell line [7]. To model the effect of perturbation of the PI3K/PTEN/AKT signalling cycle on the whole SN, we added to the model [7] the reaction of inhibition of PI3K enzyme (see Fig. 1).

The developed model was parameterised on the basis of the experimental data on phosphorylation kinetics of HER2, ERK, AKT, and PTEN in the absence and presence of 
pertuzumab [7]. Figs. 2A and 2C show agreement between theoretical and experimental data on the phosphorylation kinetics of pHER2 and pAKT. Computational modelling of the SN was performed using the DBSolve package for kinetic modelling [23] and SimBiology modelling software (MATLAB, The MathWorks Inc.). The review of kinetic modelling approach used in the work is given in [24].

\subsubsection{Control parameter derivation}

To derive the control parameter of the PI3K/PTEN/AKT signalling pathway we analysed analytically the kinetic properties of the key subsystem of the whole $\mathrm{SN}$ - the PI3K/PTEN/AKT signalling cycle (see subsystem framed in Fig. 1). This cycle consists of two inter-convertible enzymes, phosphoinositide 3-kinase, PI3K, and phosphatase, PTEN (tensin homolog deleted on chromosome 10), as well as the protein kinase, AKT. PI3K enzyme converts phosphatidylinositol (PIP2) to phosphatidylinositol-3,4,5-trisphosphate (PIP3), and in turn PTEN dephosphorylates PIP3 and recycles the process [25]. The consumption of PIP2 and the production of the active complex AKT-PIP3 in the cycle are determined by the relationship between the activities of these enzymes.

To define the control parameter characterising the dynamics of the PI3K/PTEN/AKT cycle we considered its kinetic model and studied the steady state of this subsystem. The model includes the following reactions of the whole SN kinetic model [7] (see subsystem framed in Fig. 1):

$$
\begin{aligned}
& V_{P I 3 K}: \quad \mathrm{PIP} 2+\mathrm{PI} 3 \mathrm{~K}^{*} \underset{k_{-1}}{\stackrel{k_{1}}{\leftrightarrow}} \mathrm{PI} 3 \mathrm{~K}^{*}-\mathrm{PIP} 2 \stackrel{k_{c a t, P I 3 K}}{\rightarrow} \mathrm{PI} 3 \mathrm{~K}^{*}+\mathrm{PIP} 3 ;
\end{aligned}
$$

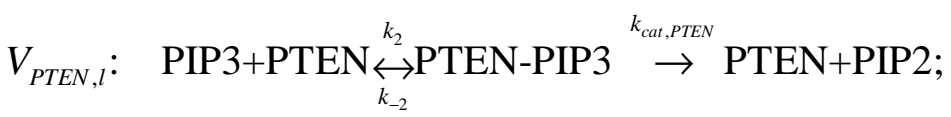

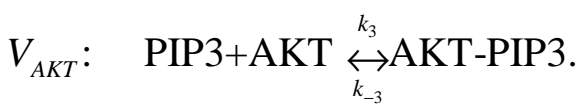

Analysing the steady state of the systems of ordinary differential equations (ODEs) corresponding to the model (1)-(3) (see Appendix), one can derive a relationship between the steady state concentrations of the substrate, $P I P 2_{s}$, the end product, $A K T-P I P 3_{s}$, of the cycle and the enzymes, $P I 3 K_{s}^{*}, P T E N_{s}$ as:

$$
\frac{K_{m, P I 3 K}}{k_{c a t, P I 3 K} P I 3 K_{s}^{*}} \frac{k_{c a t, P T E N} P T E N_{s}}{K_{m, P T E N}} \frac{K_{d, A K T}}{A K T_{o}}=P I P 2_{s}\left(\frac{1}{A K T-P I P 3_{s}}-\frac{1}{A K T_{o}}\right) \text {, }
$$


where $K_{m, i}, k_{c a t, i}, K_{d, i}=k_{-i} / k_{i}-$ Michaelis, catalytic, and dissociation constants; $k_{i}$ and $k_{-i}-$ reaction rate constants of forward and reverse reactions (1)-(3).

For an approximate analysis of equation (4), we assume that the steady state concentrations of the $P T E N_{s}$ and $P I 3 K_{s}^{*}$ enzymes differ slightly from their initial concentrations, $P T E N_{o}, P I 3 K_{o}[5]$ and determine a dimensionless parameter $\Gamma$ equal to the relation of the left hand of equation (4):

$$
\Gamma=\frac{K_{m, P I 3 K}}{k_{c a t, P I 3 K} P I 3 K_{o}} \frac{k_{c a t, P T E N} P T E N_{o}}{K_{m, P T E N}} \frac{K_{d, A K T}}{A K T_{o}}=\frac{v_{P T E N}}{v_{P I 3 K} v_{A K T}} .
$$

The parameter $\Gamma$ determines the balance of the enzyme activities of PTEN, PI3K*, and AKT: $v_{\mathrm{PTEN}}, v_{\mathrm{PI} 3 \mathrm{~K}}$, and $v_{A K T}$, where

$$
v_{P T E N}=\frac{k_{c a t, P T E N} P T E N_{o}}{K_{m, P T E N}} ; \quad v_{P I 3 K}=\frac{k_{c a t, P I 3 K} P I 3 K_{o}}{K_{m, P I 3 K}} ; \quad v_{A K T}=\frac{K_{d, A K T}}{A K T_{o}} .
$$

Determined on the basis of the PI3K/PTEN/AKT cycle dynamics, $\Gamma$ was used to study the response kinetics of the whole SN to RTK activation and inhibition. It is clear that the complex dynamics of the large-scale signal transduction system cannot be described completely by a single parameter, but we suggest that some key features of its functioning may be controlled by the abstracted parameter $\Gamma$. Note that a similar parameter was introduced by Ventura et al. [22] to analyse the dynamics of separate phosphorylation/dephosphorylation cycles and their cascades in MAPK pathway.

$\Gamma$ can be changed as a result of the following cellular processes: 1) enzyme inhibition causing a decrease in the effective concentration of enzyme, unbound with inhibitor; 2) variation in enzyme expression level leading to changes in enzyme concentration; and 3) enzyme mutations resulting in possible alterations of the catalytic constant, $k_{\text {cat }}$ and/or enzyme affinity to the substrate, $K_{d}$ (or Michaelis constant, $K_{m}$ ). Throughout our analyses, we use the normalized parameter $\gamma$ where $\gamma=\Gamma / \Gamma_{0}$. The value of $\Gamma_{o}$ corresponds to the reference enzyme activities of PTEN, PI3K, and AKT used in the modelling of pertuzumab effect [7] $\left(\Gamma_{\mathrm{o}}\right.$ $=3 \cdot 10^{4}$ ). This allows us to explore the relative impact of proportional changes in $\gamma$, with $\gamma=1$ corresponding to a normal response to RTK inhibition. Note that the control parameter $\gamma$ is the extension of the resistance factor [7], which considers the balance of the activities only of two enzymes: PI3K and PTEN.

\subsection{Experimental methods}

\subsubsection{Cell Culture and Collection of Lysates}


PE04 cells were routinely grown as monolayer cultures in DMEM supplemented with $10 \%$ heat-inactivated fetal calf serum (FCS) and penicillin / streptomycin (100IU/mL) in a humidified atmosphere of $5 \% \mathrm{CO}_{2}$ at $37^{\circ} \mathrm{c}$. Time course experiments were set up by plating cells into $10 \mathrm{~cm}$ petri dishes, which were then left for $48 \mathrm{~h}$. Cells were washed briefly in PBS before transfer to phenol red-free DMEM containing 5\% double charcoal-stripped serum supplemented with penicillin/streptomycin $(100 \mathrm{IU} / \mathrm{mL})$ and glutamine $(0.3 \mathrm{mg} / \mathrm{mL})$ for a further $48 \mathrm{~h}$ prior to treatment to ensure estrogen free conditions. Paired lysates were prepared by first treating relevant dishes with pertuzumab $(100 \mathrm{nmol} / \mathrm{L})+/-\mathrm{bpV}($ pic $)(50 \mathrm{nmol} / \mathrm{L})+/-$ LY294002 (5 $\mu \mathrm{mol} / \mathrm{L})$ immediately followed by the addition of heregulin- $\beta$ ( $1 \mathrm{nmol} / \mathrm{L})$. Samples were collected at time points of 1, 2, 5, 10, 30, and 60 min for dynamic time course profiles, or $60 \mathrm{~min}$ for static measurements, washed in PBS, and immediately lysed in icecold isotonic lysis buffer [50 mM Tris- $\mathrm{HCl}(\mathrm{pH} 7.5), 5 \mathrm{mM}$ EGTA (pH 8.5), $150 \mathrm{mM} \mathrm{NaCl}$, $1 \%$ Triton X-100] supplemented with aprotinin $(10 \mu \mathrm{g} / \mathrm{mL})$ and a protease inhibitor cocktail (Roche, 11836153001). Lysates were centrifuged for $6 \mathrm{~min}$ at 13,000 $\mathrm{x} \mathrm{g}$ and protein concentrations of supernatants subsequently determined using the BCA assay (Sigma, BCA$1)$.

\section{Western Blotting}

Protein lysates were electrophoretically resolved on either $10 \%$ or $12 \%$ SDS-PAGE and transferred overnight onto Immobilon-P membranes (Millipore, Bedford, MA). After transfer, membranes were blocked with $1 \%$ blocking agent (Roche, \#1520709) in TBS before probing overnight at $4{ }^{\circ} \mathrm{C}$ with the appropriate primary antibody made up in $0.5 \%$ blocking agent. Primary antibodies used for western blotting were as follows: anti-phospho-AKT $\left(\operatorname{Ser}^{473}\right)$ (Cell Signaling Technology, \#9271) at 1:1000; anti-AKT (Cell Signaling Technology, \#9272) at 1:1000; anti-phospho-p44/42 MAP Kinase $\left(\mathrm{Thr}^{202} / \mathrm{Tyr}^{204}\right.$ ) (Cell Signaling Technology, \#9101) at 1:1000; anti-p44/42 MAP Kinase $\left(\mathrm{Thr}^{202} / \mathrm{Tyr}^{204}\right.$ ) (Cell Signaling Technology, \#9102) at 1:1000; anti-HER1 (Cell Signaling Technology, \#2232) at 1:1000; anti-HER2 (Cell Signaling Technology, \#2242) at 1:1000; anti-HER3 (Cell Signaling Technology, \#4754) at 1:1000; anti-HER4 (Cell Signaling Technology, \#4795) at 1:1000; anti-PTEN (Cell Signaling Technology, \#9552) at 1:1000; anti-phospho-PTEN (Ser ${ }^{370}$ ) (Upstate, \#07-889) at 1:1000. Immunoreactive bands were detected using enhanced chemiluminescent reagents (Roche, 1520709) and Hyperfilm ECL film (GE Healthcare, UK). Bands were scanned using an Epson Perfection 4990 scanner and Integrated Optical Density (IOD) absorbance values were 
obtained by densitometric analysis using Labworks gel analysis software (UVP Life Sciences, Cambridge, UK).

\section{Results}

\subsection{The effect of yon signalling response to RTK inhibition}

We focussed on the sensitivity of the AKT response as a surrogate readout of the SN to changes in the control parameter of PI3K/PTEN/AKT signalling cycle, $\gamma$. To analyse the dependence of AKT activation on $\gamma$ we compared the theoretical response kinetics of the system to inhibition of input signal, pHER2, simulated at different values of $\gamma$, where this input signal is both independent on $\gamma$ and agrees with experimental data (see Fig. 2A). The system response at $\gamma=1$ showed that $90 \%$ inhibition of pHER 2 resulted in approximately $90 \%$ and $80 \%$ inhibition effect on PI3K and AKT activation respectively at 60 min after HRG stimulation (see Figs. 2B and 2C).

In our model formulation, decreasing $\gamma$ had no impact on the response of PI3K activation, as $\gamma$ controls the downstream PI3K/PTEN/AKT cycle kinetics (the results shown in Fig. 2B are independent of $\gamma$ ). However, the output signal pAKT was sensitive to the value of $\gamma$. Fig. 2D shows the kinetics of the pAKT signal in the absence and presence of pertuzumab calculated for a low value of $\gamma ; \gamma=0.5$. As can be seen from Fig. 2D there is a loss in sensitivity to treatment, with no inhibition effect from $100 \mathrm{nM}$ pertuzumab on pAKT signal under this condition: the concentration of pAKT remains unchanged in the presence of pertuzumab after $30 \mathrm{~min}$ of HRG stimulation. Thus, in contrast to the case where $\gamma=1$ (Fig. 2C), the inhibition of RTK and PI3K (Figs. 2A and 2B) does not cause inhibition of the pAKT signal (Fig. 2D). This kinetics may be explained by amplification of a small, inhibited RTK signal into a maximal output signal, pAKT, by the PI3K/PTEN/AKT cycle at low values of $\gamma$. Below we analyse this amplification regime of the signalling cycle.

To show in detail how the PI3K/PTEN/AKT cycle controls the level of output signal, pAKT, we have calculated the consumption kinetics of PIP2, for the different pHER2 signals at $\gamma=1$ and 0.5 (see Figs. 2E and 2F). As can be seen, the steady state concentration of PIP2 depends on pertuzumab presence as well as $\gamma$. When $\gamma=1$, the steady state concentration of PIP2 differs in the absence and presence of pertuzumab (see Fig. 2E). This difference of the two signals translates into a difference in AKT activation signals (see Fig. 2C). In contrast, at 
$\gamma=0.5$ there is little distinction between PIP2 concentrations in the absence and presence of pertuzumab after 30 min of HRG stimulation (see Fig. 2F). This small difference does not lead to a system-scale difference between the two final pAKT signals, despite the presence of RTK inhibitor (see Fig. 2D). Note that in the model we do not consider cellular metabolism of PIP2 [26] and its effect on the signalling, neglecting de novo synthesis and membrane diffusion of PIP2 during signal generation.

This result shows interconnection between $\gamma$ and the compensatory properties of the PI3K/PTEN/AKT signalling network against inhibition of receptor signal. This compensatory mechanism thus confers insensitivity of AKT activation signal to inhibition of receptor stimuli. This compensatory effect on the reduction of $\gamma$ results from the transition of the system from sensitivity to resistance regime, which in itself arises from the perturbation of the balance among enzyme activities in the PI3K/PTEN/AKT cycle.

To analyse this effect we calculated the pAKT dose-dependence on pertuzumab concentration for different values of $\gamma$ (see Fig. 3). The black line in Fig. 3 presents the pAKT dose-dependence calculated for the reference case $\gamma=1$. According to this result, the obtained value of pertuzumab $\mathrm{IC}_{50}$ is equal to $40 \mathrm{nM}$, in agreement with known experimental data [7]. To model the pAKT dose-dependence at $\gamma<1$ we have taken into account in the model the inhibition of PTEN enzyme by the bpV(pic) inhibitor [27]. PTEN inhibition leads to a decrease in $\gamma$ to $\gamma<1$ due to a fall in the effective concentration of enzymes, unbound with inhibitor. Note that we demonstrate below that both PI3K and AKT activities may also be varied to change $\gamma$. The results of the calculation showed that the pAKT dose-dependence at $\gamma<1$ (blue line in Fig. 3 ) is transformed to a higher pertuzumab concentrations $\left(\mathrm{IC}_{50}=1 \mu \mathrm{M}\right)$. This leads to resistance of AKT activation at pertuzumab concentrations in the region of the typical $\mathrm{IC}_{50}(40 \mathrm{nM})$ for this drug.

The theoretical prediction of the resistance acquisition against RTK inhibition at the loss of PTEN activity has been confirmed by experiment with PTEN inhibition by the $\mathrm{bpV}$ (pic) inhibitor [7]. Fig. 4 shows a comparison of the theoretical and experimental data on phosphorylated AKT concentration observed at 30 min after HRG activation of the human ovarian carcinoma cell line PE04 at different combinations of inhibitors. Bars 3 show an inhibition effect of pertuzumab on AKT phosphorylation. The inhibition of PTEN by $\mathrm{bpV}$ (pic) leads to compensation for pertuzumab action (see bars 5 in Fig. 4) and restores the initial level of HGR-activated AKT signal (see bars 2 in Fig. 4). Note that PTEN inhibitor $\mathrm{bpV}$ (pic) does not influence AKT activation when added alone (see bars 8 in Fig. 4). Thus the 
change of the systems parameter of the PI3K/PTEN /AKT cycle, $\gamma$, as a result of PTEN inhibition, leads to the sensitivity-to-resistance transition in the SN response to RTK inhibition.

\subsection{Analysis of the sensitivity-to-resistance transition}

To study the sensitivity-to-resistance transition in the PI3K/PTEN/AKT pathway in detail, we calculated the dependence of the output signal, pAKT(pHER2, $\gamma$ ), on the input signal, pHER2 for different values of $\gamma$ (see Fig. 5). The amplitude of the pHER2 signal was varied by changing HER2 inhibitor concentration and this amplitude was normalised to the maximal value of the pHER2 signal in the absence of pertuzumab. The different values of $\gamma$ correspond to different initial concentrations of PTEN enzyme, while the activities of PI3K and AKT are assumed constant (see equation (5)). Below we also show that a similar dependence, pAKT(pHER2, $\gamma$ ), may be obtained by likewise varying PI3K or AKT enzyme activities.

The smooth dependence pAKT(pHER2, $\gamma$ ) at the values of $\gamma \cong 1$ shows that the signalling system was sensitive with respect to the level of input signal, that is the output pAKT signal was nonlinearly proportional to the input signal, pHER2 (see line 6 in Fig. 5). In this sensitivity regime the PI3K/PTEN/AKT cycle functions as a discriminator of the receptor signal, i.e. under this condition, inhibition of HER2 receptor caused a corresponding inhibition of pAKT signal.

When $\gamma<1$, as a result of the loss of PTEN activity (see equation (5)), there was a dramatic change in the PI3K/PTEN/AKT cycle behaviour (see lines 1-5 corresponding to $\gamma<1$ in Fig. 5). At these values of $\gamma$ the cycle switches to hypersensitivity (amplification) mode with near zero-order kinetics $[17,18]$. Beginning with a definite threshold of pHER2, the system amplifies the input pHER2 signals up to the maximal output pAKT signal. In this case, the lower the value of $\gamma$, the weaker the HER2 signal that is amplified. In this regime, the signalling network response becomes insensitive to the level of external signals, i.e., the output signal does not depend on the input signal for a wide range of pHER2 values (for example, from 0.05 to 1 at $\gamma=0.5$, see line 2 in Fig. 5). We suggest that this amplification regime corresponds to resistance of pAKT response to RTK inhibition and the transition from sensitivity to resistance regime represents a compensatory mechanism of the SN. 
The increase in $\gamma$ to values higher than 1 caused suppression of pAKT signal, relative to the reference point $\gamma=1$, everywhere over the region of pHER 2 signal values (see line 7 corresponding to $\gamma>1$ in Fig. 5). In this region of $\gamma$, the PI3K/PTEN/AKT cycle inhibits pAKT signalling response and switches to suppression mode.

The sensitivity-to-resistance transition was also observed when changes in $\gamma$ represent variation in PI3K or AKT enzyme activity with constant activities of both PTEN and AKT or PI3K respectively. Supplementary Fig. 1 shows the dependence of pAKT(pHER2, $\gamma$ ) when $\gamma$ changes due to variation of dissociation constants of PI3K and AKT enzymes. According to the definition of $\gamma$ (see equation (5)), increasing PI3K activity through decreasing of the dissociation constant of PI3K, $K_{d, P I 3 K}$, leads to a decrease in $\gamma$ and therefore to the sensitivityto-resistance transition in the SN. Supplementary Fig. 1A shows that pHER2 signals higher than 0.2 are amplified up to the maximal pAKT signal. The similar behaviour in the dependences of pAKT(pHER2, $\gamma$ ) was observed in the model at the decrease in control parameter $\gamma$ due to the increase of the dissociation constant of AKT, $K_{d, A K T}$ (see Supplementary Fig. 1B).

Using these results, and to explore the impact of changes in activities of PTEN, PI3K and AKT on resistance of the $\mathrm{SN}$, we defined the resistance index $\mathrm{R}$ (as in [7]) of the $\mathrm{SN}$ as the value of the pAKT signal at a specified level of the RTK inhibition. Choosing $90 \%$ inhibition of the pHER2 signal (see Fig. 2A) we introduced the resistance index, $\mathrm{R}_{90}$, as the ratio $\mathrm{R}_{90}=\mathrm{pAKT}_{90} / \mathrm{pAKT}_{0}$, where $\mathrm{pAKT}_{90}$ is the value of the $\mathrm{pAKT}$ signal where $90 \%$ of the pHER2 signal is inhibited and $\mathrm{pAKT}_{0}$ is the pAKT signal in the absence of RTK inhibition. $\mathrm{R}_{90}$ reflects the change in responsiveness, or resistance, of the $\mathrm{SN}$ to the inhibition of receptor signals. Fig. 6 shows the theoretical dependence of the resistance index $R_{90}(\gamma)$ on $\gamma$, where $\gamma$ was varied by changing PTEN, PI3K or AKT enzyme activities. Loss of PTEN activity exerted the most pronounced effect on the resistance index $R_{90}(\gamma)$ (black line in Fig. 6).

Maximal resistance, when $\mathrm{R}_{90} \cong 1$, means that $90 \%$ inhibition of RTK causes no inhibition of the pAKT signal. When $\gamma$ was varied through loss of PTEN activity, the maximal resistance index $R_{90}(\gamma)$ was observed at $\gamma<0.6$. In this region of $\gamma$, the PI3K/PTEN/AKT cycle amplifies the inhibited RTK signals up to the maximal value and thereby compensates for inhibition of the input signals. Thus, at these values of $\gamma$ the SN manifests resistance against HER2 inhibition. On increasing $\gamma$, the resistance index $\mathrm{R}_{90}(\gamma)$ decreased, and the resistance-tosensitivity transition occurred. In the region $\gamma \cong 1$, the $\mathrm{SN}$ was sensitive to input signals: $90 \%$ 
RTK inhibition causing $~ 80 \%$ inhibition of the pAKT signal $\left(\mathrm{R}_{90}(\gamma=1)=0.2\right)$. Variation of PI3K and AKT activities exerted a weaker influence on the resistance index $\mathrm{R}_{90}(\gamma)$ (see blue and red lines in Fig. 6).

The sharp transition in $\mathrm{R}_{90}(\gamma)$ on varying PTEN activity arises from post-translational regulation of PTEN (see black line in Fig. 6). It is known that activation of PTEN is controlled by the phosphorylation/dephosphorylation cycle, where the phosphorylated form of PTEN is inactive [25]. In our model of PTEN [7] we have taken into account the PTEN phosphorylation/dephosphorylation cycle (see PTEN/pPTEN cycle in Fig. 1), with the phosphorylation reaction phenomenologically describing phosphorylation of PTEN by casein kinase 2 (CK2) and glycogen synthase kinase $3 \beta$ (GSK3) [25]. As it is unknown which enzyme dephosphorylates PTEN [25], we assumed a weak protein phosphatase activity of PTEN [7] and its auto-dephosphorylation properties [25]. Our calculation showed the important role of the PTEN/pPTEN cycle in the regulation of the PI3K/PTEN/AKT cycle. The deletion of this cycle from the model resulted in the disappearance of the sharp transition in the resistance index dependence, $\mathrm{R}_{90}(\gamma)$ (see dashed black line in Fig. 6), and the resistance index dependence was more similar to the cases corresponding to changes in $\gamma$ due to PI3K and AKT activity variations (blue and red lines in Fig. 6). The impact of the PTEN/pPTEN cycle on the PI3K/PTEN/AKT cycle behavior underlies the irregularities in the function pAKT $(\mathrm{pHER} 2, \gamma)$ visible in Fig. 5. This threshold transition from sensitivity to resistance at the decrease of PTEN activity results from additional inactivation of PTEN due to its phosphorylation by CK2 and GSK3, which in turn causes the decrease of $\gamma$.

$\gamma>1$ leads to a decrease in the resistance index $\operatorname{R}_{90}(\gamma)$, meaning that the PI3K/PTEN/AKT cycle causes additional suppression of the inhibited RTK signal (corresponding to the suppression region at $\gamma>1$ in Fig. 6). High values of $\gamma$ can be observed in cells in response to increasing of PTEN activity due to gene amplification [12], or the decreasing of PI3K activity at its inhibition [28], or the reduction in AKT activity as a result of its inhibition or mutation [29].

\subsection{The effects of combination inhibition on the sensitivity-to-resistance transition}


The combined effect of RTK and PTEN inhibitors (pertuzumab and bpV(pic), respectively) discussed in section 3.1 showed that PTEN inhibition abrogates the inhibition effect of pertuzumab. Here, the additional effect of the PI3K inhibitor, LY294002 [28] is considered individually and then in combination with RTK and PTEN inhibitors (Fig. 1).

First, we calculated pAKT dose-dependence on LY294002 concentration (see black line in Fig. 7). At the concentration of LY294002 near its $\mathrm{IC}_{50}(4 \mu \mathrm{M})$ the effective concentration of PI3K* active form reduces by a factor of six (data are not shown), leading to $\gamma$ increasing from 1 to 6, and the PTEN/PI3K/AKT cycle to function in the suppression regime. The experimental measurement of pAKT inhibition confirms the model prediction of $60 \%$ pAKT inhibition at $5 \mu \mathrm{M}$ of LY294002 at $30 \mathrm{~min}$ after HRG stimulation (see bars 7 in Fig. 4 and the point at the black line in Fig. 7).

Second, we considered the combined effect of PTEN and PI3K inhibition on pAKT dose-dependence. As can be seen in the definition of $\gamma(5)$, the inhibition of PTEN and PI3K affects the parameter $\gamma$ in opposite directions: that is PTEN inhibition causes a decrease in $\gamma$ and a move of the SN function to the resistance region; and PI3K inhibition leads to an increase in $\gamma$ that shifts the system into the suppression region. We calculated the pAKT dosedependence on LY294002 concentration in the presence of PTEN inhibitor, bpV(pic) (see dotted line in Fig. 7). The action of bpV(pic) causes a shift of the pAKT dose-dependence to higher concentrations of LY294002 relative to that calculated for the PI3K inhibitor alone (solid line in Fig. 7). Thus, PTEN inhibition leads to suppression of the PI3K inhibitor action in the wide concentration range near the $\mathrm{IC}_{50}$ of LY294002. Here, PTEN inhibition compensates for pAKT signal inhibition by PI3K inhibitor, and the PTEN inhibitor plays an antagonist role against the PI3K inhibitor. This PTEN-induced resistance to PI3K inhibition is similar to PTEN-induced resistance to RTK inhibition discussed in section 3.1. Note, that the theoretically obtained ten times shift of $\mathrm{IC}_{50}$ together with the change in shape of the pAKT dose-dependence for LY294002 at PTEN inhibition by bpV(pic) (see Fig. 7) is in agreement with experimental data [27].

We next considered the effect of another combination of two inhibitors: PI3K inhibitor, LY294002, and RTK inhibitor, pertuzumab (see scheme in Fig. 1). Fig. 3 shows the theoretical pAKT dose-dependence for pertuzumab in the presence of LY924002 (green line). As can be seen, PI3K inhibitor leads to a shift of the dose-dependence and pertuzumab $\mathrm{IC}_{50}$ towards lower concentrations of pertuzumab relative to that calculated for pertuzumab alone (black line in Fig. 3). Thus, the PI3K inhibitor is synergistic when combined with the RTK 
inhibitor. The increase in $\gamma$ resulting from the PI3K inhibition (see equation (5)) causes the SN transition towards the suppression regime.

Summarising the effect of $\gamma$ on AKT activation, Fig. 3 shows the pAKT dosedependences for the RTK inhibitor (pertuzumab), calculated at different values of the parameter $\gamma: \gamma=1$ (pertuzumab alone, black line); $\gamma<1$ (pertuzumab $+b p V($ pic), blue line); and $\gamma>1$ (pertuzumab+ LY294002, green line). The observed shifts of the pertuzumab IC $_{50}$ confirm the effect of each inhibitor on $\gamma$ and the behaviour of the PTEN/PI3K/AKT cycle.

In addition to these pairwise effects, we considered the effect of both inhibitors LY294002 and bpV(pic) on RTK inhibition. As discussed above, the loss of PTEN activity as a result of PTEN inhibition by bpV(pic) leads to compensation for the effect of RTK inhibition by pertuzumab (see blue line in Fig. 3). This compensation effect is due to the sensitivity-to-resistance transition occurring when $\gamma<1$. The additional inhibition of PI3K in this case will cause $\gamma$ to return to the initial value $\gamma \cong 1$. We hypothesised this restores responsiveness to RTK inhibition by reversing the sensitivity-to-resistance transition.

To verify this we calculated the pAKT dose-dependence for pertuzumab in combination with LY294002+bpV(pic) (see red line in Fig. 3). As can be seen, the addition of PI3K inhibitor causes a shift in the pAKT dose-dependences calculated at PTEN inhibition to the position close to the dose-dependences obtained for pertuzumab alone (black line in Fig. 3). The approximate matching of the two dose-dependencies simulated without/with LY294002+bpV(pic) combination (black and red lines) confirms that PI3K and PTEN inhibitors compensate for one another at $\gamma \cong 1$.

To test the model predictions experimentally, we have ascertained the effects of LY294002 alone and the LY294002+bpV(pic) combination on pertuzumab inhibition in the ovarian cancer cell line PE04. The measurements were performed at the inhibitor concentrations corresponding to the character points given at the theoretical dose-dependence curves (see Figs. 3 and 7). Comparison of the theoretical and experimental results is shown in Fig. 4. Bars 6 depict the combined effect of LY294002 and bpV(pic) on pertuzumab action. The experimental data confirms the predicted effect of mutual compensation for two inhibitor action, LY294002 and bpV(pic) and restoration of pertuzumab inhibition. The comparison of inhibition effects of LY294002 alone (bars 7) and in combination with pertuzumab (bars 4) shows the synergetic action of RTK and PI3K inhibitors, as predicted in the model. 
The comparison of the overall theoretical and experimental data depicted in Fig. 4 shows that the proposed model of SN response control [7] describes satisfactorily the individual effects of three inhibitors as well as their combination actions.

\section{Discussion}

A systems biology approach was employed to study the compensatory mechanisms underlying the observed counteraction of the signalling network to inhibition of RTK in human cancer cell lines. We focussed on the compensatory mechanisms arising from the functioning of a key building block involved in the PI3K/AKT signalling pathway - the PI3K/PTEN/AKT cycle. This cycle, and the enzymes involved in it, have been found to play a crucial role both in tumorigenesis and acquisition of resistance to cancer therapy [1,13,29-38]. Using kinetic modelling, combined with experimentation, we showed that the PI3K/PTEN/AKT cycle can implement a compensatory mechanism against RTK inhibition. The results indicated that the system-level properties of the whole SN depend upon the balance among activities of the enzymes involved in the PI3K/PTEN/AKT cycle.

To explore these properties, we introduced a control parameter $\gamma$, encapsulating the ratio of the enzyme activities $v_{P T E N}: v_{P I 3 K}: v_{A K T}$ (see equation (5)) and studied the response of the whole system to heregulin-induced activation and RTK inhibition at different values of $\gamma$. The results of the modelling showed that the $\mathrm{SN}$ can function in three different modes and transition between these modes can occur by varying $\gamma$ : 1) sensitivity $(\gamma \cong 1), 2)$ resistance $(\gamma<1)$, and 3) suppression $(\gamma>1)$. In sensitivity mode inhibition of the input signal, pHER2, caused inhibition of the output signal, pAKT. In resistance mode the system is robust to RTK inhibition, since this inhibition does not influence the saturated level of AKT activation in a wide physiological range of inhibitor concentrations. In suppression mode, the system was found to inhibit receptor signals.

The introduction of $\gamma$ permits the analysis, in a unified fashion, of the effects of enzyme activity imbalance in the PI3K/PTEN/AKT cycle which could be caused by possible mutations and/or inhibition of the enzymes involved. We considered loss of PTEN activity, observed in various cancer types due to PTEN mutation [13,32-34] and shown to contribute to the acquired resistance to RTK inhibitors $[7,33]$. Both the theoretical and experimental results obtained confirmed that loss of PTEN activity, leading to a decrease in $\gamma$, induces the sensitivity-to-resistance transition and causes resistance to RTK inhibition [7]. We also 
considered the effect of changing $\gamma$ as a result of a kinetic property variation of PI3K enzyme as well as its inhibition by LY294002. The mutations of PIK3CA gene leading to the catalytic activation of PI3K have been found to occur in different types of cancer [35-37] and also to introduce resistance to RTK inhibition [13]. Modelling showed that activation of PI3K enzyme due to an increase in its affinity to substrate leads to an increase in $\gamma$ and moves the SN response into the resistance region. Likewise, the effect of a rise of AKT enzyme activity on imbalance in the PI3K/PTEN/AKT cycle was modelled. The increasing of AKT affinity to the lipid second messenger, PIP3 decreases $\gamma$ and switches the system function into the resistance mode. Such perturbation of the PI3K/PTEN/AKT cycle as a result of AKT overproduction and aberration in its expression were observed in different types of cancer $[13,29,38,39]$.

As an illustration of the key role of $\gamma$ in the resistance of the SN to RTK inhibition we represent in Fig. 8 our experimental data on the correlation between $\gamma$ and the inhibition of growth rate of 13 ovarian carcinoma cell lines [7]. Here, $\gamma$ was estimated based on Western Blotting data on the total concentrations of PTEN, PI3K, and AKT enzymes as well as the assessment of the four most common mutations in the PIK3CA gene [7,37]. These mutations were assumed to increase the activity of PI3K twofold [38]. As the kinetic properties of these enzymes as well as the expression status of other proteins involved in the $\mathrm{SN}$ were not considered in the calculation, this result has to be regarded as an approximation. Despite this, the obtained dependence demonstrates a notable correlation between the balance of enzyme activities in PI3K/PTEN/AKT cycle and the inhibition of growth rate for various ovarian cancer cell lines. This shows that $\gamma$ may be a useful indicator in determining the efficacy of pertuzumab for distinct cancer cell lines.

The results of the modelling demonstrate that it is possible to reverse resistance-tosensitivity transition by changing $\gamma$. Resistance against receptor inhibition can be aborted for a certain range of RTK inhibitor concentration by external perturbation of the PI3K/PTEN/AKT cycle. To study this, we simulated the effects of combinations of two and three inhibitors and carried out complementary experiments on combination inhibition of HRG-induced activation of human ovarian carcinoma cell line PE04. Results show that resistance against RTK inhibition acquired as a result of the partial loss of PTEN activity $(\gamma<1)$ may be restored by increasing $\gamma$ up to 1, e.g. due to PI3K inhibition (see red line in Fig. 3). We propose that this effect is observed in the case of cancer cells with PTEN mutation because of the non-zero level expression of PTEN detected in these cancer cells [40]. We demonstrate that 
perturbation of the enzyme activity balance in the PI3K/PTEN/AKT cycle is likely to be responsible for both cancer development (robust tumour growth) and acquired and de novo resistance against RTK inhibition (cancer robustness). The most pronounced effect on resistance was loss of PTEN activity, and this is determined by the interaction between PI3K/PTEN/AKT cycle and post-translation regulation of PTEN activity in the PTEN/pPTEN cycle, governed by CK2/GSK3 enzymes as well as auto-dephosphorylation activity of PTEN [25].

From our investigations into the combined action of inhibitors, we propose that targeting different enzymes involved in compensatory loops is a promising approach to prevent and suppress resistance against RTK inhibition. Consequently, combined theoretical and experimental studies of the compensatory mechanisms of the SN against external perturbations may inform development of effective combination cancer therapy [41].

\section{Conclusions}

We developed a computational and experimental approach to dissect the compensatory mechanisms underlying resistance of the PI3K/PTEN/AKT signalling network to RTK inhibition. We demonstrated that the compensatory properties of the $\mathrm{SN}$ may be characterised by a system-level control parameter, $\gamma$ (generalised resistance factor [7]), which may be estimated experimentally from protein abundance and enzyme kinetic property data.

We studied the compensatory loops involving PTEN, PI3K, AKT, and CK2/GSK3 enzymes arranged in individual modules of a large-scale RAF/ERK/PI3K/AKT signalling network. This study highlighted the key role of PTEN in the compensatory response of the SN in the transition from sensitivity to resistance to RTK inhibition in combination treatment regimes [7].

More broadly, we have shown a generalisable and scalable approach to support further unravelling of the compensatory loops including other enzymes and subsystem motifs of SNs to address drug resistance [42]. Moreover, the approach proposed here may be used to inform the identification of therapeutic biomarkers. This is made possible by the systematic manipulation of signalling network dynamics through key control parameters that govern the dynamics of the whole signalling network combined with a mechanistic interpretation of that manipulation, enabling model exploration to inform directly the drug discovery process. 


\section{Appendix}

The system ODEs corresponding to submodel of PI3K/PTEN/AKT cycle (see framed module in Fig. 1 and reactions (1)-(3)) is represented in the form

$$
\begin{array}{lll}
\frac{d P I P 2}{d t}=-V_{1}+V_{-1}+V_{c a t, P I 3 K} & \text { (A1) } & \frac{d P I 3 K^{*}}{d t}=-V_{1}+V_{-1}+V_{c a t, P I 3 K} \\
\frac{d P I P 3}{d t}=-V_{2}+V_{-2}+V_{c a t, P I 3 K}-V_{3}+V_{-3} & \text { (A2) } & \frac{d P T E N}{d t}=-V_{2}+V_{-2}+V_{c a t, P T E N} \\
\frac{d P I 3 K-P I P 2}{d t}=-V_{1}+V_{-1}-V_{c a t, P I 3 K} & \text { (A3) } & \frac{d A K T}{d t}=-V_{3}+V_{-3} \\
\frac{d P T E N-P I P 3}{d t}=V_{2}-V_{-2}-V_{c a t, P T E N} & \text { (A4) } & \frac{d A K T-P I P 3}{d t}=V_{3}-V_{-3},
\end{array}
$$

where the reaction rates $V_{i}$ are defined by the following rate equations in action mass approximation

$$
\begin{array}{lll}
V_{1}=k_{1} \cdot P I 3 K * \cdot P I P 2 & V_{2}=k_{2} \cdot P I P 3 \cdot P T E N & V_{3}=k_{3} \cdot P I P 3 \cdot A K T \\
V_{-1}=k_{-1} \cdot P I 3 K-P I P 2 & V_{-2}=k_{-2} \cdot P T E N-P I P 3 & V_{-3}=k_{-3} \cdot A K T-P I P 3 \\
V_{c a t, P I 3 K}=k_{c a t, P I 3 K} \cdot P I 3 K-P I P 2 & V_{c a t, P T E N}=k_{c a t, P T E N} \cdot P T E N-P I P 3 &
\end{array}
$$

For system (A1)-(A8) the following conservation lows occur

$A K T+A K T-P I P 3=A K T_{o} ; P I 3 K^{*}+P I 3 K-P I P 2=P I 3 K_{o}^{*} ; P T E N+P T E N-P I P 3=P T E N_{o}$

$P I P 2+P I P 3+A K T-P I P 3+P I 3 K-P I P 2+P T E N-P I P 3=P I P 2_{o}$

where $A K T_{o}, P I 3 K_{o}^{*}, P T E N_{o}$, and $P I P 2_{o}$ are total concentrations of the species.

From the relations of equilibrium states of PI3K-PIP2 ${ }_{\mathrm{s}}\left(\mathrm{A} 3\right.$ ) and PTEN-PIP3 ${ }_{\mathrm{s}}(\mathrm{A} 4)$ one can derive steady state concentrations of enzyme-substrate complexes PI3K-PIP2 $2_{s}$, PTENPIP3 $3_{\mathrm{s}}$ and final complex AKT-PIP3s:

$$
\begin{aligned}
& P I 3 K-P I P 2_{s}=\frac{P I P 2_{s} \cdot P I 3 K_{s}^{*}}{K_{m, P I 3 K}}, P T E N-P I P 3_{s}=\frac{P I P 3_{s} \cdot P T E N_{s}}{K_{m, P T E N}}, \\
& A K T-P I P 3_{s}=\frac{A K T_{s} \cdot P I P 3_{s}}{K_{d, A K T}},
\end{aligned}
$$

where

$$
K_{m, P I 3 K}=\frac{k_{c a t, P I 3 K}+k_{-1}}{k_{1}}=\frac{k_{c a t, P I 3 K}}{k_{1}}+K_{d, P I 3 K}, \quad K_{m, P T E N}=\frac{k_{c a t, P T E N}+k_{-2}}{k_{2}}=\frac{k_{c a t, P T E N}}{k_{2}}+K_{d, P T E N}
$$

are Michaelis constants and $K_{d, P I 3 K}, K_{d, P T E N}$, and $K_{d, A K T}-$ dissociation constants. 
Substituting equations (A10) into equilibrium relation of PIP2 (A1), one can obtain the relationship between steady sate concentrations of $\mathrm{PI} 3 \mathrm{~K}_{\mathrm{s}}^{*}, \mathrm{PTEN}_{\mathrm{s}}, \mathrm{PIP} 2_{\mathrm{s}}$, and PIP3 $3_{\mathrm{s}}[18]$ as

$$
\frac{K_{m, P I 3 K}}{k_{c a t, P I 3 K} P I 3 K_{s}^{*}} \frac{k_{c a t, P T E N} P T E N_{s}}{K_{m, P T E N}}=\frac{P I P 2_{s}}{P I P 3_{s}} .
$$

Expressing the concentration of PIP $3_{\mathrm{s}}$ in term of AKT-PIP3 ${ }_{\mathrm{s}}$ concentration (A10) and using the conservation low for AKT (A9) we can rewrite relation (A11) as

$$
\frac{K_{m, P I 3 K}}{k_{c a t, P I 3 K} P I 3 K_{s}^{*}} \frac{k_{c a t, P T E N} P T E N_{s}}{K_{m, P T E N}} \frac{K_{d, A K T}}{A K T_{o}}=P I P 2_{s}\left(\frac{1}{A K T-P I P 3_{s}}-\frac{1}{A K T_{o}}\right) \text {. }
$$

\section{Acknowledgments}

This work was supported by grants from Breakthrough Breast Cancer, the Breast Cancer Campaign, BBSRC and the Scottish Funding Council (SRDG). 


\section{References}

[1] A. Citri, Y. Yarden, Nat. Rev. Mol. Cell. Biol. 7 (2006) 505-516.

[2] M. Hatakeyama, S. Kimura, T. Naka, T. Kawasaki, N. Yumoto, M. Ichikawa, J.H. Kim, K. Saito, M. Saeki, M. Shirouzu, S. Yokoyama, A. Konagaya, Biochem. J. 373 (2003) 451-463.

[3] M.R. Birtwistle, M. Hatakeyama, N. Yumoto, B.A. Ogunnaike, J.B. Hoek, B.N. Kholodenko, Mol. Syst. Biol. 3 (2007) 144.

[4] W.W. Chen, B. Schoeberl, P.J. Jasper, M. Niepel, U.B. Nielsen, D.A.Lauffenburger, P.K. Sorger, Mol Syst Biol 5 (2009) 239.

[5] B. Schoeberl, E.A. Pace, J.B. Fitzgerald, B.D. Harms, L. Xu, L. Nie, B. Linggi, A. Kalra, V. Paragas, R. Bukhalid, V. Grantcharova, N. Kohli, K.A. West, M. Leszczyniecka, M.J.

Feldhaus, A.J. Kudla, U.B. Nielsen, Sci. Signal. 2 (2009) ra31.

[6 ] C.S. Park, I.C. Schneider, J.M. Haugh, J. Biol. Chem. 278 (2003) 37064-37072.

[7] D. Faratian, A. Goltsov, G. Lebedeva, A. Sorokin, S. Moodie, P. Mullen, C. Kay, I. H. Um, S. Langdon, I. Goryanin, D.J. Harrison, Cancer Res. 69 (2009) 6713-620.

[8] R.P. Araujo, L.A. Liotta, E.F. Petricoin, Nat. Rev. Drug Discov. 6 (2007) 871-880.

[9] H. Kitano, Mol. Syst. Biol. 3 (2007) 137.

[10] J. Stelling, U. Sauer, Z. Szallasi, F.J. Doyle 3rd, J. Doyle, Review Cell 118 (2004) 67585.

[11] H. Kitano, Nat. Rev. Drug Discov. 6 (2007) 202-220.

[12] H. Kitano, Nat. Rev. Cancer 4 (2004) 227-235.

[13] J.A. McCubreya, L.S. Steelmana, Advan. Enzyme Regul. 46 (2006) 249-279.

[14] C.F. Singer, W.J. Köstler, G. Hudelist, Biochim. Biophys. Acta 1786 (2008) 105-113.

[15] P. Mullen, D.A. Cameron, M. Hasmann, J.F. Smyth, S.P. Langdon, Mol. Cancer Ther. 6 (2007) 93-100.

[16] A. Kong, V. Calleja, P. Leboucher, A. Harris, P.J. Parker, B. Larijani, PLoS ONE 3 (2008) e2881.

[17] E.R. Stadtman, P.B. Chock, Proc. Natl. Acad. Sci. USA 74 (1977) 2761-2765.

[18] A. Goldbeter, D.E. Koshland Jr, Proc. Natl. Acad. Sci. USA 78 (1981) 6840-6844.

[19] C. Gomez-Uribe, G.C. Verghese, L.A. Mirny, PLoS Comput. Biol. 3 (2007) 2487-2497.

[20] N.I. Markevich, J.B. Hoek, B.N. Kholodenko, J. Cell Biol. 164 (2004) 353-359.

[21] H. Shankaran, D.L. Ippolito, W.B. Chrisler, H. Resat, N. Bollinger, L.K. Opresko, H.S. Wiley, Mol. Syst. Biol. 5 (2009) 332.

[22] A.C. Ventura, J.A. Sepulchre, S.D. Merajver, PLoS Comput. Biol. 4 (2008) e1000041. 
[23] I. Goryanin, T.C. Hodgman, E. Selkov, Bioinformatics 15 (1999) 749-758.

[24] O. Demin, I. Goryanin, Kinetic Modelling in Systems Biology. Chapman and Hall/CRC, 2008 .

[25] N.R. Leslie, C.P. Downes, Biochem J. 382 (2004) 1-11.

[26] L.M. Loew, J. Physiol. 582 (2007) 945-951.

[27] A.C. Schmid, R.D. Byrne, R. Vilar, R. Woscholski, FEBS Lett. 566 (2004) 35-38.

[28] C.J. Vlahos, W.F. Matter, K.Y. Hui, R.F. Brown, J. Biol. Chem. 269 (1994) 5241-5248.

[29] A. Noske, A. Kaszubiak, W. Weichert, C. Sers, S. Niesporek, I. Koch, B. Schaefer, J.

Sehouli, M. Dietel, H. Lage, C. Denkert, Cancer Lett. 246 (2007) 190-200.

[30] A.M. Al-Khouri, Y. Ma, S.H. Togo, S. Williams, T. Mustelin, J. Biol. Chem. 80 (2005) 35195-35202.

[31] A. Silva, J.A. Yunes, B.A. Cardoso, L.R. Martins, P.Y. Jotta, M. Abecasis, A.E. Nowill, N.R. Leslie, A.A. Cardoso, J.T. Barata, J. Clin. Inves. 118 (2008) 3762-3774.

[32] K. Berns, H.M. Horlings, B.T. Hennessy, M. Madiredjo, E.M. Hijmans, K. Beelen, S.C. Linn, A.M. Gonzalez-Angulo, K. Stemke-Hale, M. Hauptmann, R.L. Beijersbergen, G.B. Mills, M.J. van de Vijver, R. Bernards, Cancer Cell 12 (2007) 395-402.

[33] Y. Nagata, K.H. Lan, X. Zhou, M. Tan, F.J. Esteva, A.A. Sahin, K.S. Klos, P. Li, B.P. Monia, N.T. Nguyen, G.N. Hortobagyi, M.C. Hung, D. Yu, Cancer Cell 6 (2004) 117-127. [34] S. Lee, E.J. Choi, C. Jin, D.H. Kim, Gynecol. Oncol. 97 (2005) 26-34.

[35] L. Shayesteh, Y. Lu, W.L. Kuo, R. Baldocchi, T. Godfrey, C. Collins, D. Pinkel, B. Powell, G.B. Mills, J.W. Gray, Nat. Genet. 21 (1999) 99-102.

[36] D.A. Levine, F. Bogomolniy, C.J. Yee, A. Lash, R.R. Barakat, P.I. Borgen, J. Boyd, Clin. Cancer. Res. 11 (2005) 2875-2878.

[37] J.D. Carson, G. Van Aller, R. Lehr, R.H. Sinnamon, R.B. Kirkpatrick, K.R. Auger, D. Dhanak, R.A. Copeland, R.R. Gontarek, P.J. Tummino, L. Luo, Biochem. J. 409 (2008) 519524.

[38] A. Burchert, Y. Wang, D. Cai, N. von Bubnoff, P. Paschka, S. Müller-Brüsselbach, O.G. Ottmann, J. Duyster, A. Hochhaus, A. Neubauer, Leukemia 19 (2005) 1774-1782.

[39] C. Page, H.J. Lin, Y. Jin, V.P. Castle, G. Nunez, M. Huang, J. Lin, Anticancer Res. 20 (2000) 407-416. 
[40] R.T. Abbott, S. Tripp, S.L. Perkins, K.S. Elenitoba-Johnson, M.S. Lim, Mod. Pathol. 16 (2003) 607-612.

[41] J.B. Fitzgerald, B. Schoeberl, U.B. Nielsen, P.K. Sorger, Nat. Chem. Biol. 2 (2006) 458466.

[42] B.N. Rexer, J.A. Engelman, C.L. Arteaga, Cell Cycle 8 (2009) 18-22. 


\section{Figure legends}

Fig. 1. Scheme of RAF/MEK/ERK and PI3K/PTEN/AKT signalling network. A subsystem of PI3K/PTEN/AKT cycle is marked by dotted frame. LY294002 and bpV(pic) are inhibitors of PI3K and PTEN, respectively. HER2, HER3 - the epidermal growth factor receptors; HRG heregulin; 2C4 - pertuzumab; HER23 - HER2/HER3 heterodimer; Shc - Src-homology and collagen domain protein; GS - Grb2-SOS complex; PIP2 - phosphatidylinositol; PIP3 phosphatidylinositol-3,4,5-trisphosphate; RAF* and PI3K* - activated RAF and PI3K enzymes; PP2A - protein phosphatase 2A; PDK - phosphoinositide-dependent kinase 1; MKP3 - MAPK phosphatase 3.

Fig. 2. Kinetics of pHER2 (A), PI3K* (B), pAKT (C, D) and PIP2 (E, F) induced by $1 \mathrm{nM}$ HRG in the absence (lines 1) and presence of $100 \mathrm{nM} 2 \mathrm{C} 4$ (lines 2). D and F - pAKT and PIP2 kinetics, calculated in the present of PTEN inhibitor, bpV(pic) (50 nM). Concentrations of pHER2 and pAKT are normalized to their maximal concentrations. Lines - simulated kinetics, points - experimental data in the absence (filled squares) and presence of $100 \mathrm{nM}$ pertuzumab (unfilled squares).

Fig. 3. Effect of PTEN and PI3K inhibitors and their combinations on pAKT inhibition by pertuzumab. The theoretical pAKT dose-dependence for pertuzumab alone, $\gamma=1$ (black line); at the presence of PTEN inhibitor, $\mathrm{bpV}$ (pic) $(50 \mathrm{nM}), \gamma=0.3$ (blue line); at the presence of PI3K inhibitor, LY294002 (5 $\mu \mathrm{M}), \gamma=10$ (green line); at the combination of LY294002 (5 $\mu \mathrm{M})+\mathrm{bpV}$ (pic) $(50 \mathrm{nM}), \gamma \cong 1$, (red line). The pAKT concentration was taken at 30th min after HRG stimulation and normalized to its maximal concentration. The points mark the pertuzumab concentrations at which pAKT inhibition was measured.

Fig. 4. Comparison of the theoretical and experimental data on the effects of three inhibitors, pertuzumab (2C4), $100 \mathrm{nM}$; bpV(pic), $50 \mathrm{nM}$; LY294002, $5 \mu \mathrm{M}$ and their combinations on AKT activation. Grey and black bars - experimental and simulation data on pAKT concentration at $30 \mathrm{~min}$ after HRG stimulation, respectively (normalized to its maximal concentration). 
Fig. 5. Dependence of pAKT output signal on pHER2 input signal at the different values of the control parameter $\gamma$ : line $1-\gamma=0-0.3 ; 2-0.5 ; 3-0.7 ; 4-0.8 ; 5-0.9 ; 6-1 ; 7-1.3$. The concentrations of pHER2 and pAKT are normalized to their maximal concentrations.

Fig. 6. Dependence of the resistance index $R_{90}(\gamma)$ on the control parameter $\gamma$. $\gamma$ is changed by variation of the enzyme activities of PTEN (black line), PI3K (blue line), AKT (red line), and both PTEN and CK2/GSK3 (black dashed line).

Fig. 7. The pAKT dose-dependences for PI3K inhibitor (LY294002) simulated in the absence (solid line) and presence of PTEN inhibitor, $\mathrm{bpV}(\mathrm{pic}), 50 \mathrm{nM}$ (dashed line). The point marks the LY294002 concentration at which pAKT inhibition was measured. pAKT concentration is normalized to its maximal concentration.

Fig. 8. Experimental data on the correlation between the ratio $\gamma_{\exp }=\mathrm{tPTEN} /(\mathrm{tPI} 3 \mathrm{~K} \cdot \mathrm{tAKT})$ and cell growth inhibition by pertuzumab for 13 ovarian carcinoma cell lines: OAW42, OVCAR4, 59M, SKOV3, OVCAR5, PEA2, PE014, 42M, CAOV3, PE01, OVCAR3, PE04, PE06 [7]. Points - experimental data, line - regression line. 


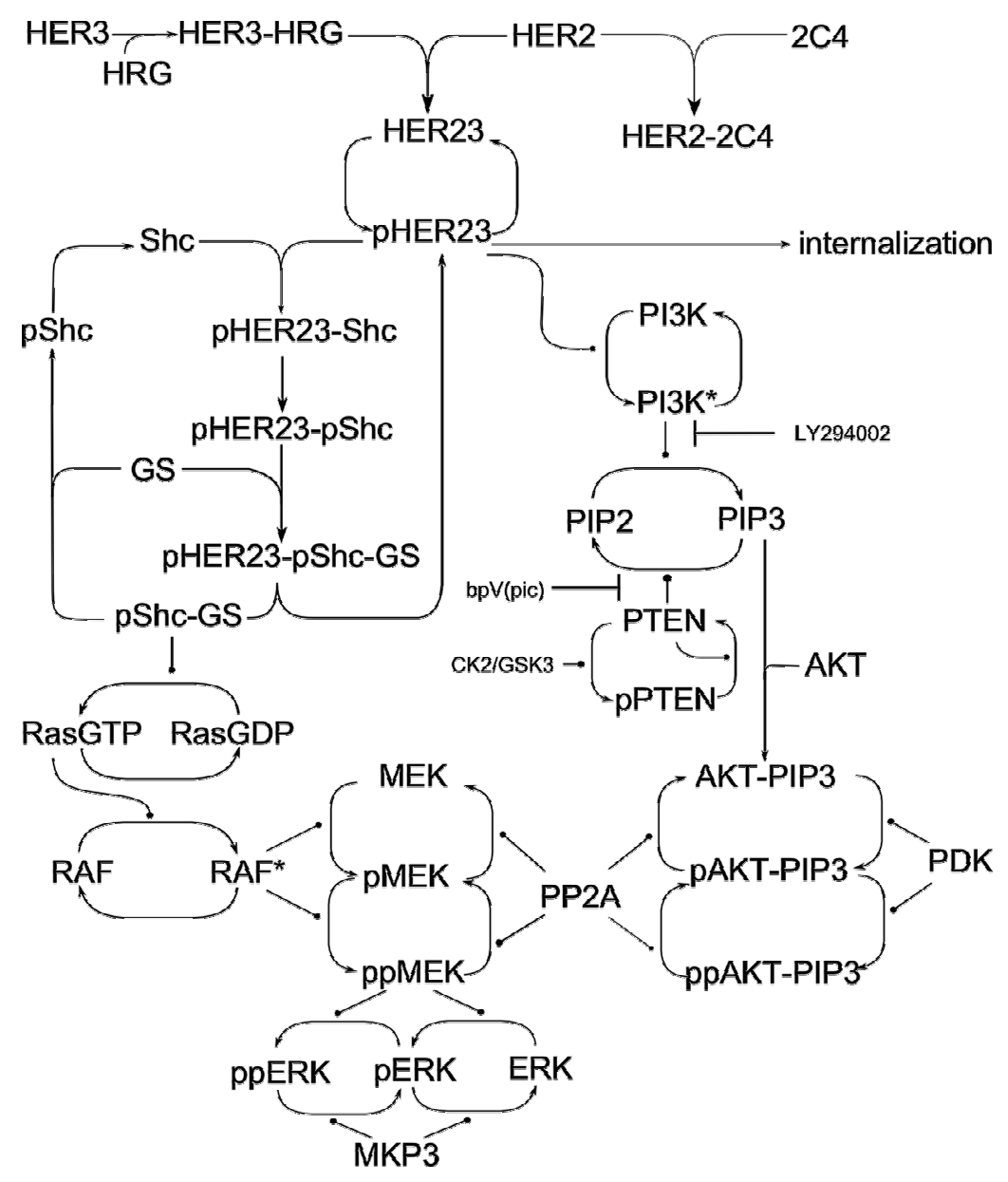

Fig. 1 
A

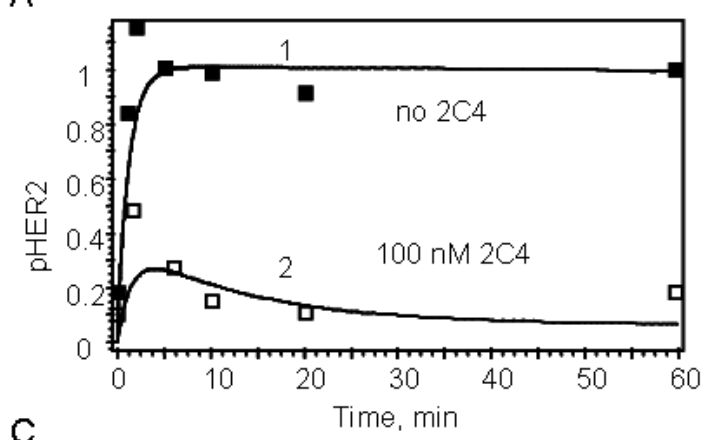

C
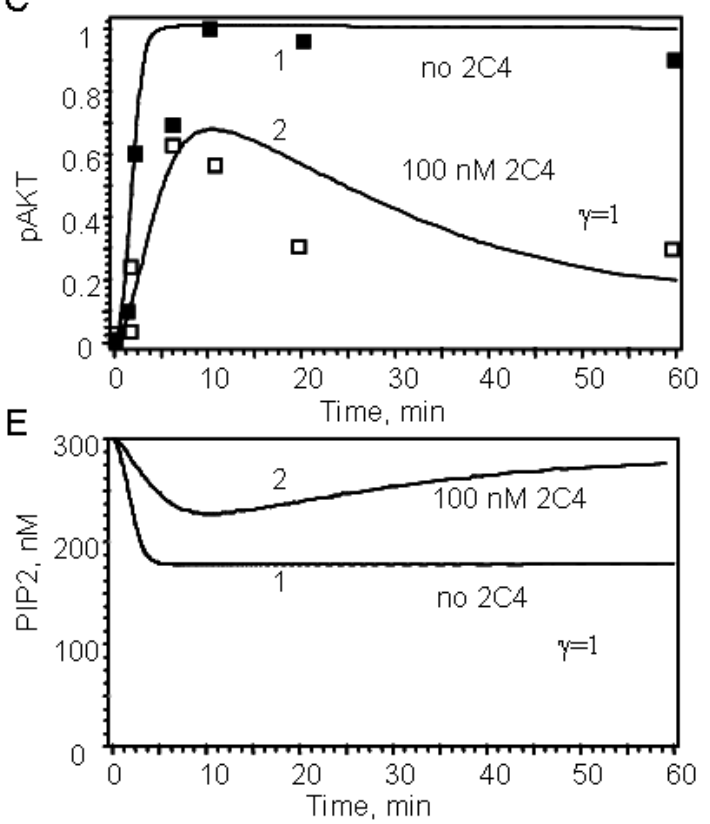

B
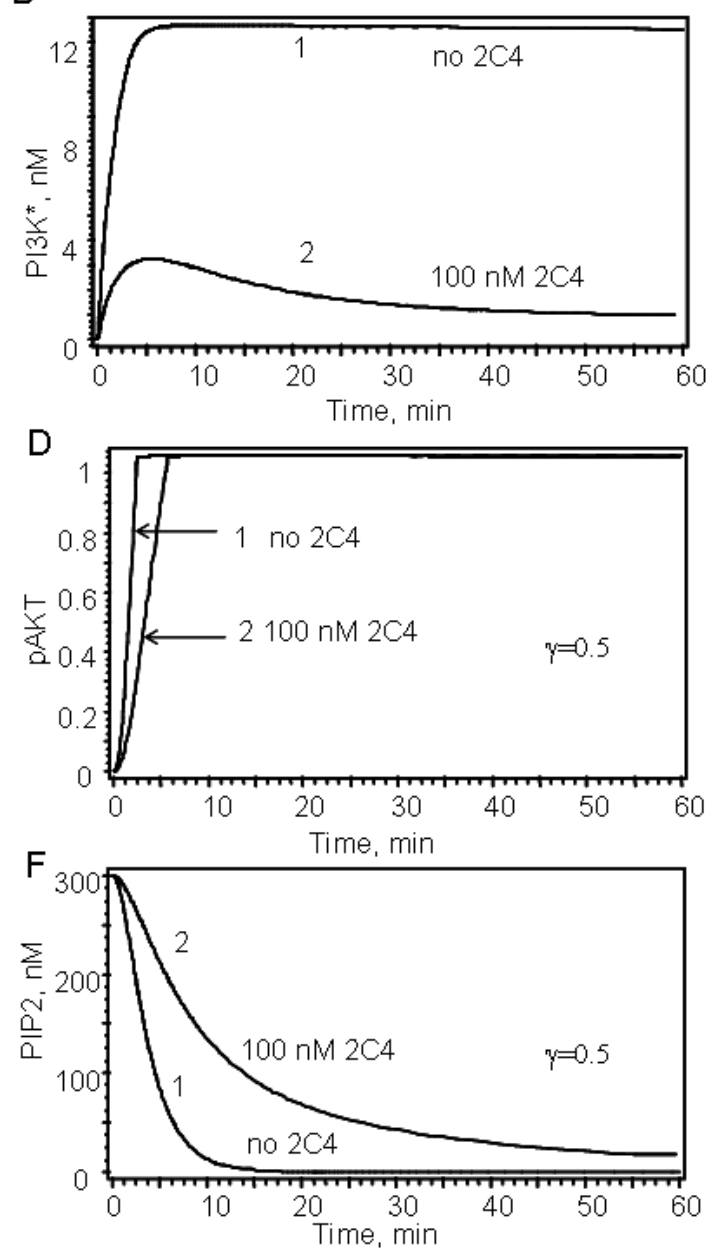

Fig. 2

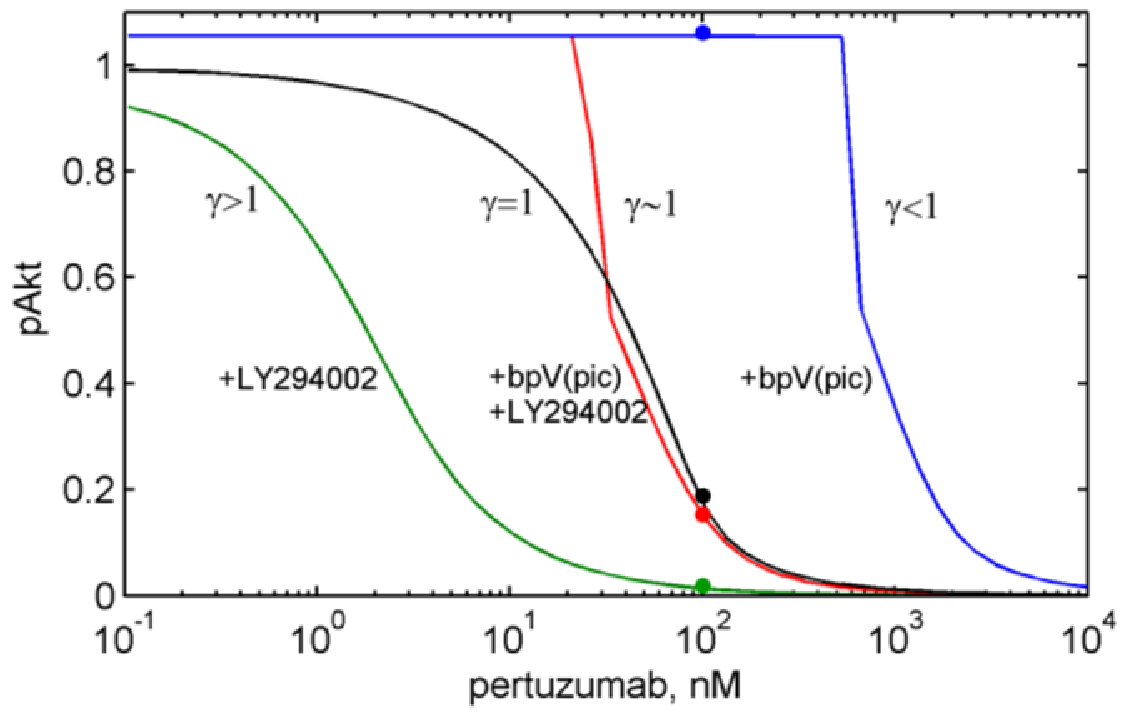

Fig. 3 


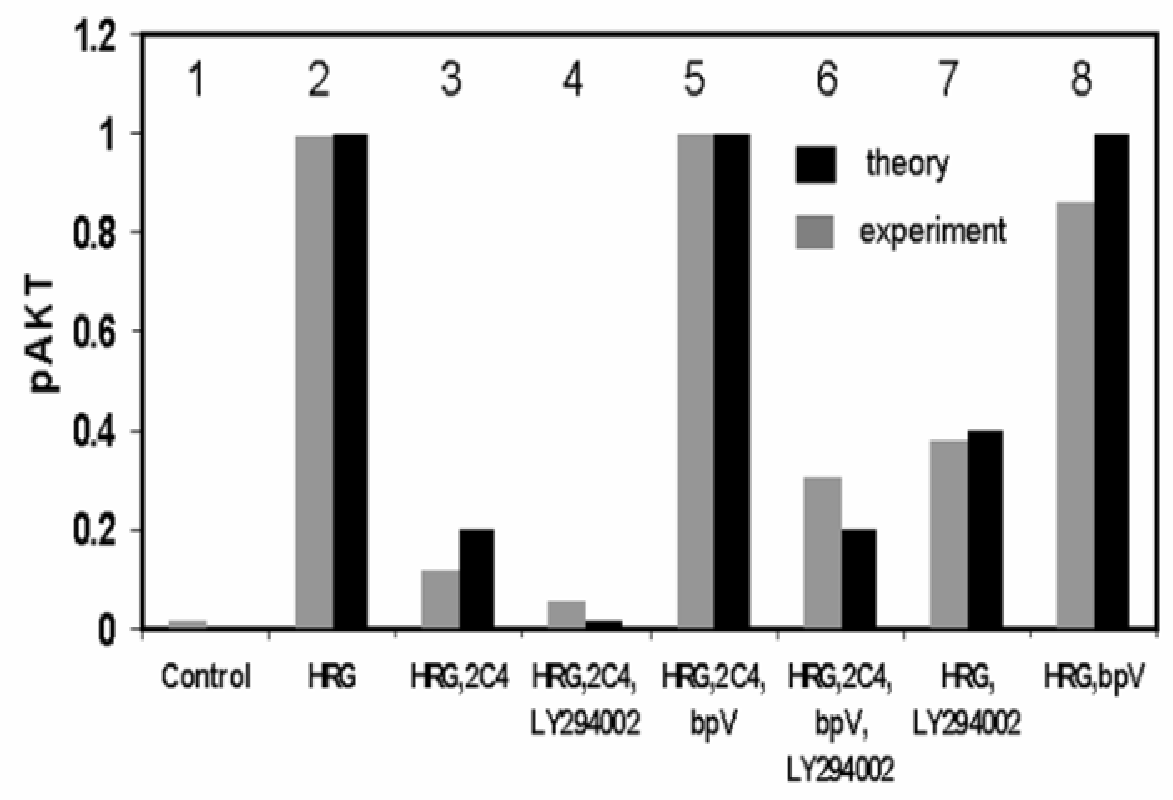

Fig. 4

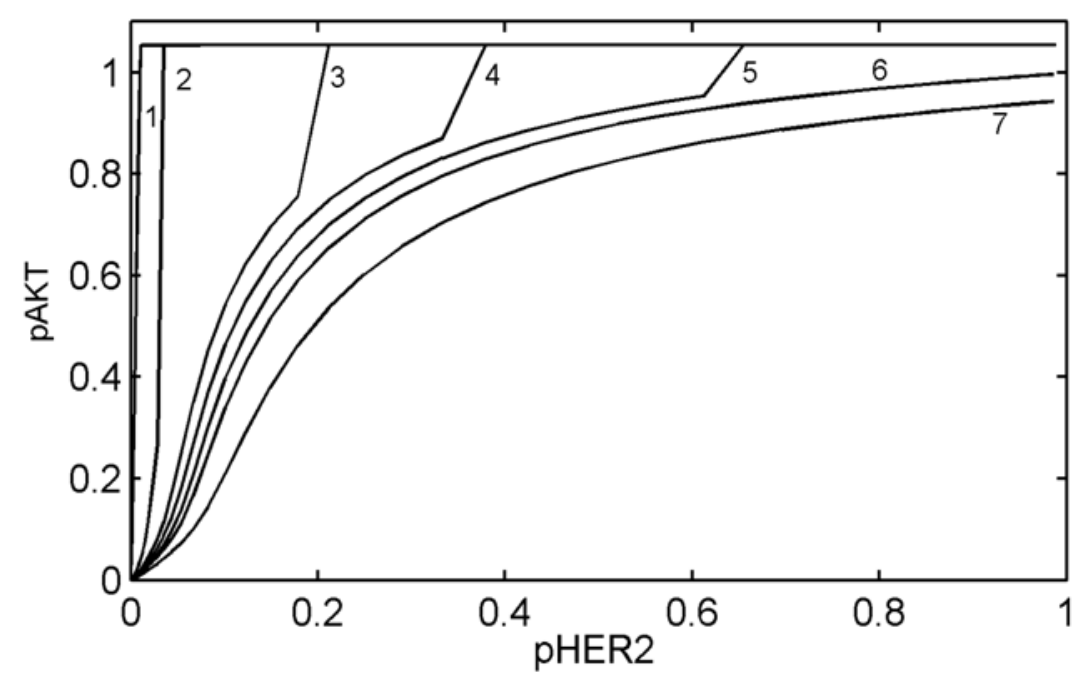

Fig. 5 


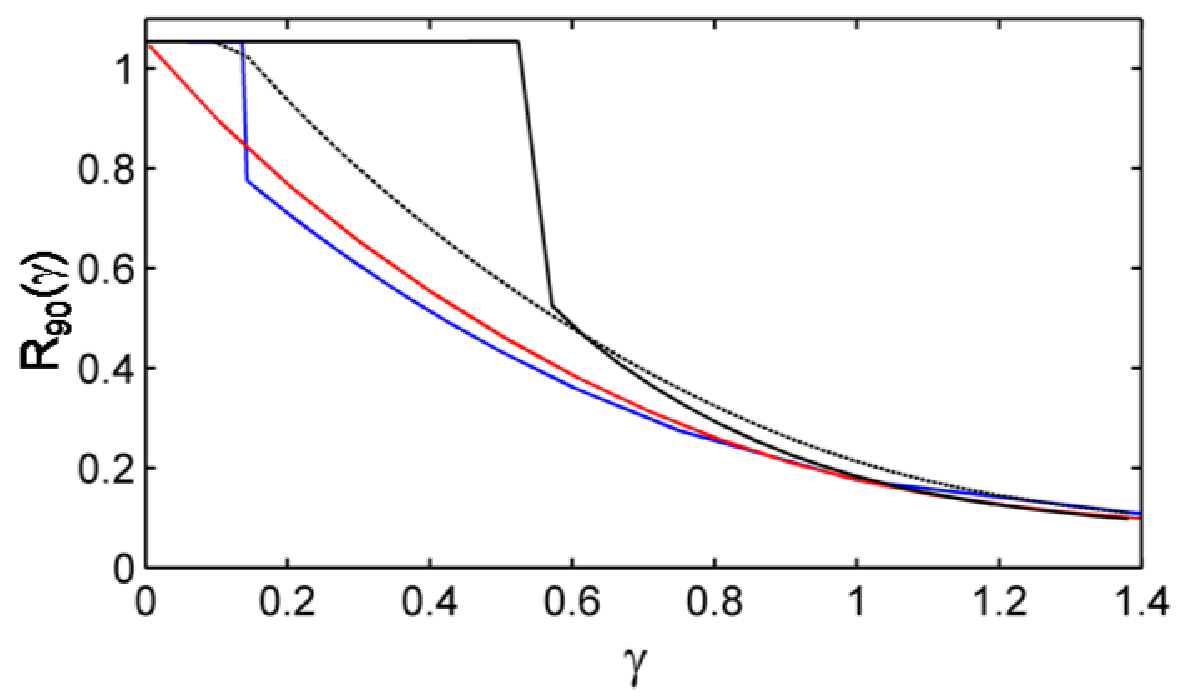

Fig. 6

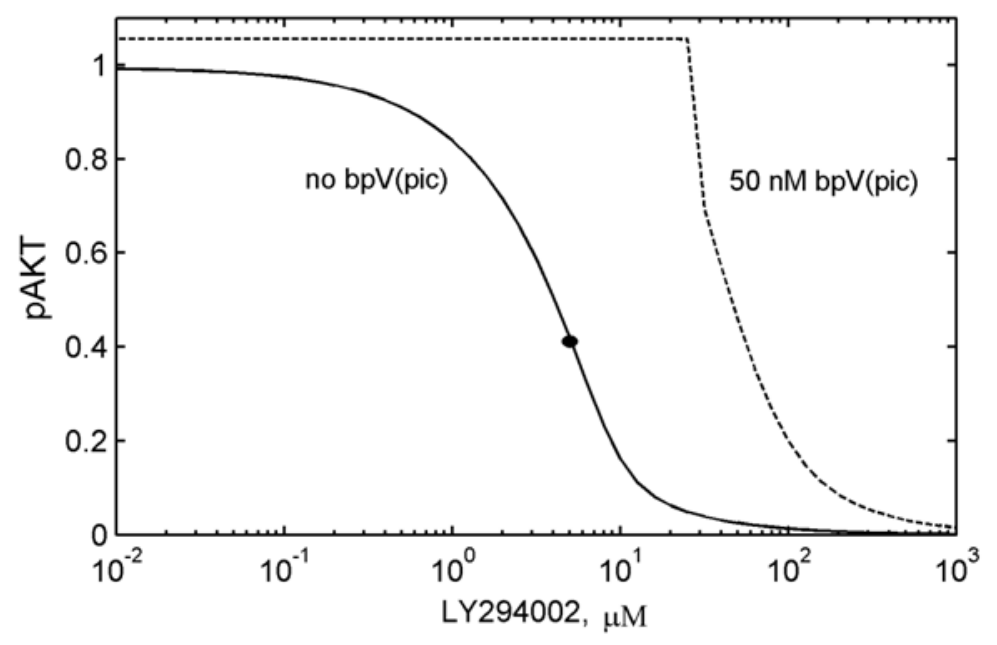

Fir. 7

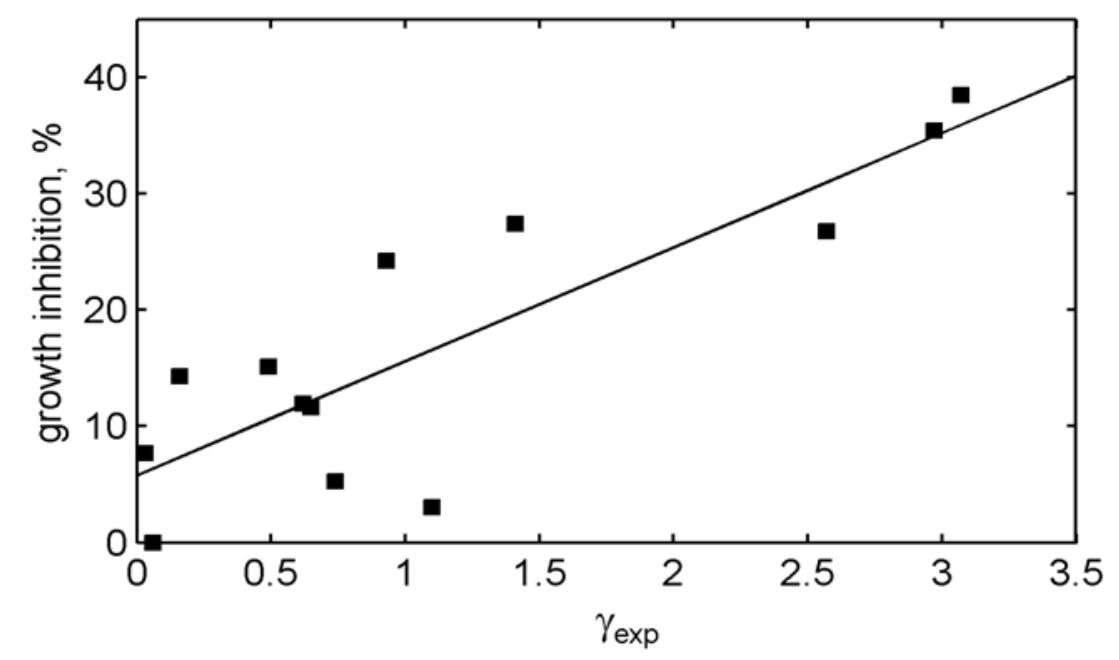

Fig. 8 


\section{Supplement. Dependence of pAKT output signal on pHER2 input signal.}

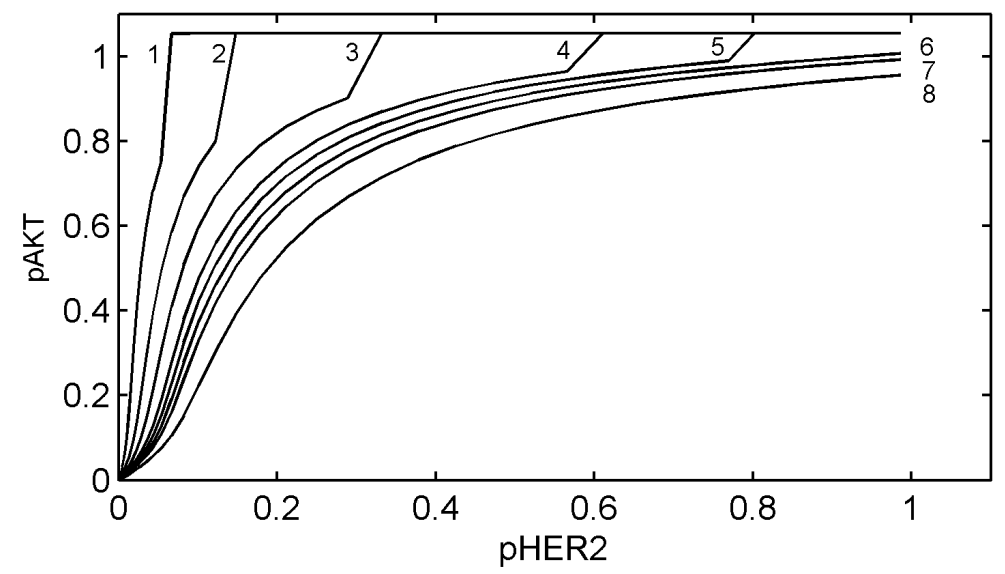

a

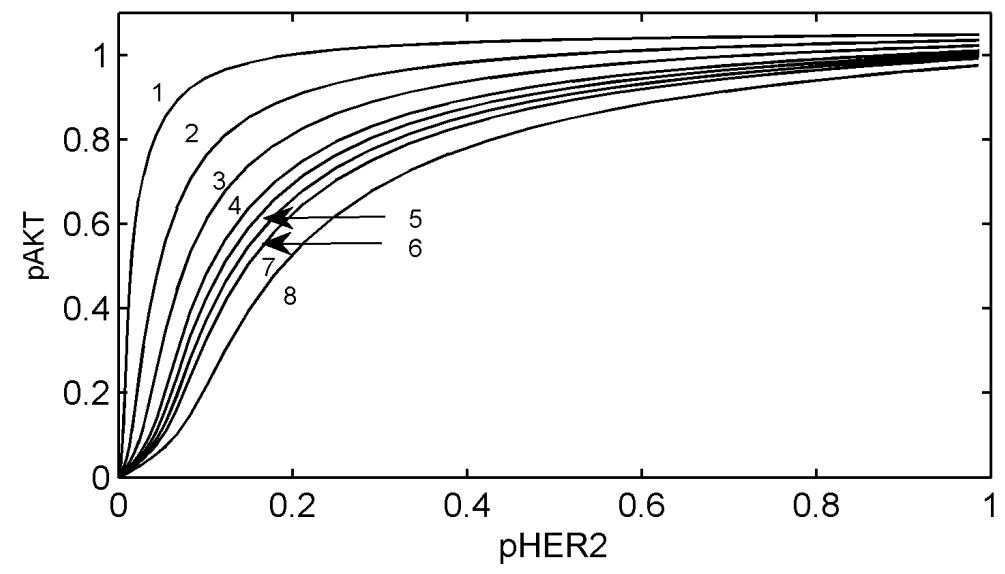

b

Supplementary Fig. 1. Dependence of pAKT output signal on pHER2 input signal at the different values of the control parameter $\gamma . \gamma$ is changed by variation of the PI3K enzyme activities (a) and affinity of AKT to PIP3 (b). $\gamma=0.1$ (line 1), 0.3 (line 2), 0.5 (line 3), 0.7 (line 4), 0.8 (line 5), 0.9 (line 6), 1 (line 7), 1.3 (line 8). 